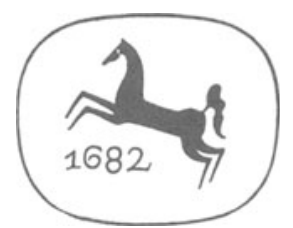

Die Geschichtlichkeit der deutschen Klassik 
Walter Müller-Seidel

\section{Die Geschichtlichkeit der deutschen Klassik}

Literatur und Denkformen um 1800

J. B. Metzlersche Verlagsbuchhandlung Stuttgart 
CIP-Kurztitelaufnahme der Deutschen Bibliothek

Müller-Seidel, Walter:

Die Geschichtlickeit der deutschen Klassik :

Literatur u. Denkformen um 1800 /

Walter Müller-Seidel.

- Stuttgart : Metzler, 1983.

ISBN 978-3-476-00529-8

ISBN 978-3-476-00529-8

ISBN 978-3-476-03177-8 (eBook)

DOI 10.1007/978-3-476-03177-8

(C) Springer-Verlag GmbH Deutschland 1983

Ursprünglich erschienen bei J. B. Metzlersche Verlagsbuchhandlung und Carl Ernst Poeschel Verlag GmbH in Stuttgart 1983 


\section{Vorwort}

Die hier vereinigten Beiträge zur Literatur der deutschen Klassik und zu ihrem geschichtlichen Verständnis wurden allesamt in den letzten fünfundzwanzig Jahren veröffentlicht: der erste (über Goethes Altersgedicht Der Bräutigam) im Jahre 1958; das Erscheinen des letzten (über Hölderlins Ode Dichterberuf in einer kürzeren Fassung) steht noch bevor. Die zunehmenende Beschäftigung mit der Literatur der Moderne in diesem Zeitraum stand diesen Arbeiten in keiner Weise im Wege. Eher das Gegenteil ist der Fall, und vielleicht ist die Hoffnung nicht ganz unberechtigt, es könnte solche Zweigleisigkeit beiden Arbeitsgebieten zugute gekommen sein. Daß man seine eigene Gegenwart nur verstehen könne, wenn man viel von Vergangenheit und Tradition versteht, ist eine eingebürgerte Weisheit unserer historischen Schulen. Sie wurde uns von der Generation unserer Lehrer eingeprägt, und wir sind mit ihr aufgewachsen. Nicht gleichermaßen eingebürgert ist die Umkehr solcher Beziehungen: die Auffassung, daß man einer Vergangenheit nur gerecht werden kann, wenn man die Gegenwart nicht aus dem Auge verliert. Zumal die Erforschung der deutschen Klassik wurde bei uns vielfach und lange Zeit in Absehung von der jeweiligen Gegenwartsliteratur betrieben. Daß mit solchen Einstellungen zumeist Formen ungeschichtlichen Denkens einhergehen, die in Ideologien einmünden können, ist in der einleitenden Betrachtung über Weimarer Klassik und Weimarer Republik ausgeführt. Deutsche Klassik und Goethezeit: das erwies sich als eine großartig dargestellte, aber auch zeitlos dargestellte Welt, in die man eingeführt wurde, wenn man im Wintersemester 1937/8 in Leipzig zu studieren begann. Die damals ungeliebte Gegenwart trug nicht wenig dazu bei, daß man in einer vergangenen Kultur wie dieser eine Art geistiger Heimat suchte - und auch fand. Sie geschichtlich zu sehen lernen, war später zu lernen. $\mathrm{Zu}$ einem nicht geringen Teil sind die hier vereinigten Arbeiten ein Niederschlag solcher Lernprozesse.

Nicht wenige Beiträge wurden Kollegen und Freunden, älteren wie jüngeren, als Beiträge zu ihren Festschriften dargebracht: Lieselotte Blumenthal, Paul Böckmann, Richard Brinkmann, Hermann Kunisch, Victor Lange, Ernest Ludwig Stahl und Benno von Wiese. Die Verbundenheit, die in solchen Widmungen zum Ausdruck gebracht wurde, besteht fort und wird mit dem veränderten Kontext, in dem diese Arbeiten nunmehr erscheinen, nur bekräftigt. Der Zeitraum dieses Vierteljahrhunderts ist zugleich derjenige, in dem das Jahrbuch der Deutschen Schillergesellschaft 
gemeinsam mit Fritz Martini und Bernhard Zeller herausgegeben wurde - als Organ zur »wissenschaftlichen Erforschung deutscher Literatur vom Beginn der Neuzeit bis zu einer der Geschichtserkenntnis bereits zugänglichen Gegenwart«, wie es im Vorwort zum erstẹn Band heißt. Eine letzte Bemerkung sei angefügt: der Hinweis, daß zwei dieser Aufsätze zuerst an Ort und Stelle der deutschen Klassik, nämlich in Weimar, vorgetragen wurden, beide aus Anlaß von Zusammenkünften der Goethe-Gesellschaft in den Jahren 1958 und 1979. Das mag man inzwischen als etwas Selbstverständliches ansehen. Aber ganz so selbstverständlich ist diese Feststellung vielleicht nicht. Solche Zusammenkünfte schließen die Möglichkeit ein, daß man die eigene Sehweise besser mit anderen vergleichen kann, als es sonst geschieht. Vor allem dienen sie dem Gespräch und einem besseren Verständnis von Wissenschaft, die noch aus anderem als nur aus dem besteht, was sich mathematisch berechnen läßt, wie eine gelegentliche Bemerkung Goethes lautet. Aus seiner Naturforschung und aus seinem Wissenschaftsbegriff sind Sprache und Gespräch nicht wegzudenken, und zumal in solchen Fragen ist von ihm noch viel zu lernen. Denn natürlich ist die Geschichtlichkeit eines geschichtlichen Phänomens immer nur die eine Seite der Sache. Die andere betrifft das, was es im Prozeß lebendiger Aneignung jeweils zu vergegenwärtigen gilt.

München, im Januar 1983

W. M. 


\section{Inhalt}

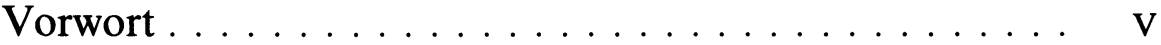

Einleitung:

Weimarer Klassik und Weimarer Republik

Zur Geschichte eines ungeschichtlichen Denkens. . . . . . . . . . . . 1

I. Das Zeitalter der Französischen Revolution . . . . . . . 31

1. Deutsche Klassik und Französische Revolution Zur Entstehung einer Denkform . . . . . . . . . . . . . 33

2. Cagliostro und die Vorgeschichte der deutschen Klassik . . . . . . . . 49

3. Auswanderungen in Goethes dichterischer Welt Zur Geschichte einer sozialen Frage . . . . . . . . . . . . . 66

II. Programmatik und Freundschaftsbund . . . . . . . . . 85

1. Schillers Kontroverse mit Bürger und ihr geschichtlicher Sinn . . . . . 87

2. Naturforschung und deutsche Klassik Die Jenaer Gespräche im Juli $1974 \ldots \ldots$. . . . . . . . . . . . . . . 105

3. Goethes Gedicht Die Braut von Korinth Zum Balladenjahr der deutschen Klassik . . . . . . . . . . . . . . . 119

III. Formen des nicht klassischen Dramas. . . . . . . . . . . . . 125

1. Die Idee des neuen Lebens in Schillers Wallenstein . . . . . . . . . . . 127

2. Episches im Theater der deutschen Klassik

Eine Betrachtung über Schillers Wallenstein . . . . . . . . . . . . 140

3. Komik und Komödie in Goethes Faust . . . . . . . . . . . . . . . . 173

IV. Gegenklassische Wendungen . . . . . . . . . . . . . . 189

1. Hölderlins Ode Dichterberuf

Zum schriftstellerischen Selbstverständnis um 1800 . . . . . . . . . . . 191

2. Kleists Penthesilea im Kontext der deutschen Klassik . . . . . . . . . . 209

3. Brentanos naive und sentimentalische Poesie . . . . . . . . . . . . 231

V. Spätwerk und Altersstil . . . . . . . . . . . . . . . . 249

1. Goethe und das Problem seiner Alterslyrik . . . . . . . . . . . . . . . 251

2. Goethes Gedicht Der Bräutigam

3. Goethes Maximen und Reflexionen

Denkformen und Bewußtseinskritik . . . . . . . . . . . . . . 274

Anmerkungen . . . . . . . . . . . . . . . . . . 287 